journal club

\title{
Ulzera heilen gut, aber Mortalität ist hoch...
}

Fragestellung: Welchen Nutzen hat eine telemedizinische Überwachung im Vergleich zur üblichen ambulanten Therapie bei Patienten mit diabetischen Fußulzera?

Hintergrund: Der Wert telemedizinischer Anwendungen in der Prävention oder der Behandlung bei diabetischen Ulzera ist, trotz einiger Studien hierzu, weiterhin unklar.

Patienten und Methoden: In dieser dänischen Studie wurde im Rahmen einer größeren europäischen Studie (Renewing Health project) der Wert telemedizinischer Anwendungen überprüft. Es wurden 736 Patienten mit diabetischen Fußulzerationen im Zeitraum von Oktober 2010 bis November 2014 gescreent, ob sie die Kriterien für diese randomisierte Studie erfüllen. Die Intervention in der Ambulanz per Telemedizin bestand nach Randomisierung aus 2 telemedizinischen Visiten, gefolgt von einem erneuten Besuch in der Fußambulanz. Die Patienten wurden von spezialisierten Krankenschwestern betreut, die bei den telemedizinischen Visiten ein Foto des Ulkus versandten, ggf. samt Wundbeschreibung online oder telefonischem Kontakt. Die Kontrollgruppe stellte sich dreimal persönlich in der Ambulanz vor. Diese 3-er Behandlungsblöcke wurden bis zur Abheilung des Ulkus wiederholt. Der Zeitabstand für die 3-er Behandlungsblöcke wurde bei jedem Patienten individuell festgelegt, entsprechend der klinischen Symptomatik. 401 Patienten erfüllten die Aufnahmekriterien, 193 wurden für die telemedizinische Betreuung und 181 für die Standardbehandlung randomisiert. Studienendpunkte waren Ulkusabheilung, Am-

\section{Originalie}

Rasmussen BSB, Froekjaer J, Bjerregaard J et al. A Randomized Controlled Trial Comparing Telemedical and Standard Outpatient Monitoring of Diabetic Foot Ulcers. Diabetes Care. 2015 38:1723-9. putation oder Tod. Die Patienten verblieben in der Studie bis einer der Endpunkte eintrat oder für maximal ein Jahr ohne eine erfolgte Ulkusabheilung.

Ergebnisse: Die mediane Studienzeit betrug 74 Tage in der Telemedizin-Gruppe und 91 Tage in der Kontrollgruppe. Die mediane Zahl der telemedizinischen Visiten betrug 3,0. Die Patientencharakteristika zu Beginn der Studie waren gleichmäßig in beiden Gruppen verteilt, das mittlere Alter der Patienten betrug 67 Jahre, die mittlere Diabetesdauer 14 Jahre. Von den 374 Patienten heilten bei 271 (72\%) die Ulzera komplett innerhalb der Studiendauer ab, ohne Unterschiede zwischen beiden Gruppen. Auch die Zahl der Amputationen unterschied sich nicht signifikant zwischen beiden Gruppen: 21 (11\%) Amputationen in der Telemedizingruppe und 26 (14\%) in der Kontrollgruppe. Auch bezüglich der Minor- und Majoramputationen fanden sich keine signifikanten Unterschiede.

Bezüglich der Mortalität fand sich jedoch ein hochsignifikanter Unterschied. Von den 9 Todesfällen (2,4\% der Patienten) traten $8(4,0 \%)$ in der Telemedizingruppe und nur einer $(0,5 \%)$ in der Kontrollgruppe auf. Nach Adjustierung für Alter, Geschlecht und Wohnort mittels des Cox proprotional Hazard Modells betrug die HR 8,68 (95\%-KI 6,93-10,88, p=0,0001).Von den 9 Todesfällen ereigneten sich 2 zu Hause, 4 im Krankenhaus und 3 auf der Intensivstation. Alle verstorbenen Patienten hatten eine chronische Herzerkrankung, 4 Personen hatten eine chronische Niereninsuffizienz und eine Person ein Prostatakarzinom. Sechs Patienten wiesen eine Sepsis auf, 5 Patienten eine Pneumonie und einer eine Gangrän.

Schlussfolgerungen: Diese bisher größte telemedizinische Studie bei Patienten mit diabetischen Fußulzera bestätigt kleinere Studien, die eine ähnlich gute Abheilung der Ulzera bei telemedizinscher Betreuung fanden. Die Autoren sehen jedoch die erhöhte Mortalität bei ihren telemedizinisch betreuten Patienten als sehr kritisch an. Aus ihrer Sicht ist dies nicht durch die Randomisierung oder andere Patientendaten zu erklären. Da Patienten mit diabetischem Fußulkus häufig eine erhebliche Komorbidität aufweisen, muss nach Ansicht der Autoren gesichert werden, dass diese Patienten besonders engmaschig überwacht werden, womit möglicherweise eine Krankenschwester mit Schwerpunkt Wundversorgung und über Telemedizin überfordert ist.

\section{- Kommentar von Prof. Dr. med. Maximilian Spraul}

\section{Hohe Komorbidität spricht für persönlichen Kontakt}

Es ist davon auszugehen, dass die Betreuung von Patienten mit diabetischen Fußulzera komplexer ist als die Betreuung von Patienten mit sonstigen Wunden, wie z.B. bei chronisch venöser Insuffizienz. Dies liegt neben den unterschiedlichen Ursachen für die Ulzera (Neuropathie, pAVK, Infektion) auch an der hohen Komorbidität der Patienten.

Möglicherweise ist die persönliche Betreuung in Fußambulanzen, bei denen der "ganze" Patient gesehen wird, für die Prognose, insbesondere der sehr kranken Patienten, günstiger.

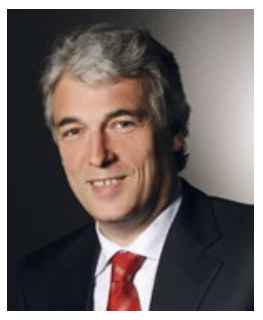

Prof. Dr. med. Maximilian Spraul

Mathias-Spital \& Jakobi-Krankenhaus Interdisziplinäres Diabetes-Fuß-Zentrum Frankenburgstr. 31 48431 Rheine m.spraul@mathias-spital.de 